\title{
From the EGR gene family to common pathways in schizophrenia: single genes versus convergent pathways
}

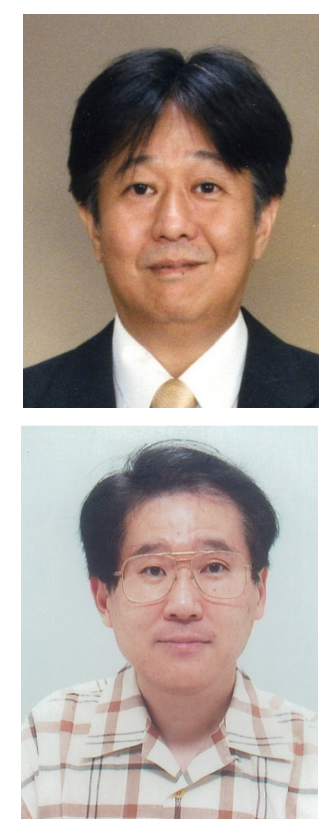

Kazuo Yamada \& Takeo Yoshikawa ${ }^{\dagger}$

${ }^{\dagger}$ Author for correspondence RIKEN Brain Science Institute, Laboratory for Molecular Psychiatry, 2-1 Hirosawa, Wako-city, Saitama 351-0198, Japan Tel.: +81484675968; Fax: +81484677462 ; takeo@brain.riken.jp

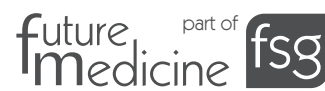

'Insight into genes associated with schizophrenia will help further our understanding of this disease and contribute to the development of new treatments.'

Schizophrenia is a devastating psychiatric disorder that afflicts one person in a hundred worldwide. It is characterized by delusions, hallucinations and disorganized behavior. The results of numerous etiological studies demonstrated that the heritability elements of schizophrenia were estimated at greater than $80 \%[1-4]$. Insight into genes associated with schizophrenia will help further our understanding of this disease and contribute to the development of new treatments. However, in keeping with other common/complex disorders, the disease shows great heterogeneity in susceptibility factors, combining multiple susceptibility genes, epigenetic processes and environmental factors that result in similar phenotypic symptoms. The discovery of genes that contribute to schizophrenia is challenging, and the cause of the disorder remains unknown. Researchers have identified several potential candidate genes for the disease, but the association results are weak and very few findings have been consistently replicated. In addition, no single proposed candidate gene is sufficient to cause the disease [3-7].

So, what is the key to solving this perplexing mystery of schizophrenia genetics? Will genetic research into schizophrenia yield positive results or end up being like a battle against windmills? New approaches in schizophrenia genetics concentrate on finding not single genes, but common pathways that potentially represent a point of convergence for molecular signaling in schizophrenia [7-9]. We recently described a novel candidate pathway that could contribute to the pathogenesis of schizophrenia. In this article, we would like to describe our strategy for finding putative molecular pathways leading to schizophrenia. For this editorial, owing to limited space, we have focused on the molecular genetic studies of schizophrenia, although there are other promising approaches. Highly plausible candidate genes, such as $B D N F$, $A K T 1$ and DISC1, have been identified through multipronged strategies. They fulfill both neurobiological and genetic criteria. Moreover, they allow for a fresh perspective and the application of novel research approaches to an old problem.

For years, schizophrenic research was based on two main pharmacological hypotheses [10]. The more established classic hyperdopaminergic hypothesis is based on the theory that antipsychotic drugs effect their therapeutic action by blocking dopamine receptors. The second, and more compelling, theory suggests a role for the hypoglutamatergic state in signal transmission.

'A recent breakthrough in the field describes the identification of candidate genes from linkage regions.'

However, genetic studies have not always confirmed a pathogenic role for dopaminergic and glutamatergic neurotransmitter genes in disease. In addition, a series of studies on genes closely related to these pathways have failed to establish an association with schizophrenia pathogenesis. These hypotheses are still attractive, but the dopaminergic and/or glutamatergic changes described may be secondary to altered signal transmission, resulting from other as yet undetermined genetic changes. To obtain a deeper understanding of the genetic determinants of this disease, a more global analysis of susceptibility genes and their causative genetic variations as a third pathway for schizophrenia is needed.

A recent breakthrough in the field describes the identification of candidate genes from linkage regions. Two strong linkage candidates for schizophrenia susceptibility are neuregulin-1 (NRG1) and dysbindin (DTNBP1). NRG1 was originally identified by the Icelandic deCODE genetics group in a genome wide linkage study and association mapping of chromosome 8p21 [11]. Neuregulin is present in glutamatergic synaptic vesicles, and affects NMDA receptors (NMDARs) via actions on ErbB receptors. DTNBP1 was isolated by association mapping across the linkage region on chromosome $6 \mathrm{p} 22.3$ 
in Irish families [12]. DTNBP1 is localized to presynaptic terminals, and may participate in the formation and maintenance of synapses. The statistical evidence for these genes is robust. Both genes regulate neurobiological roles that could contribute to disease, but the molecular mechanisms remain elusive. The accumulating evidence for these two genes raises the exciting prospect of a novel hypothesis of schizophrenia pathogenesis, which could explain their specific interactions and roles in signaling pathways.

\section{Calcineurin \& schizophrenia}

Calcineurin (protein phosphatase 2B) is another attractive candidate gene because of its function in the brain and location in the genome. It is a major calmodulin-binding protein in the brain and the only known serine/threonine protein phosphatase acting as a calcium-dependent modulator of phosphorylation status for a variety of cellular activities. It is well established that calcineurin provides a critical link between calcium regulation, synaptic plasticity, cell survival and cognition [13,14]. Interestingly, calcineurin is uniquely positioned to regulate both dopaminergic and glutamatergic signaling and integrate the activity of these two pathways $[9,15,16]$.

This protein is a heterodimer consisting of a catalytic subunit (calcineurin A; [CaNA]) and a regulatory subunit $(\mathrm{CaNB})$. There are three mammalian calcineurin catalytic isoforms, CaNA $\alpha$ (PPP3CA), $-\beta \quad(P P P 3 C B)$ and $-\gamma$ $(P P P 3 C C)$, and two regulatory isoforms, CaNB1 (PPP3R1) and CaNB2 (PPP3R2). The functional role of these CaNA subunits in the brain is not clear, but it is noteworthy that the PPP $3 C C$ subunit of CaNA is located on chromosome $8 \mathrm{p} 21.3$, one of the highlighted linkage regions for schizophrenia.

\section{'A potential mechanistic relationship between EGRs and other candidate pathways is an interesting issue for further investigation.'}

Two provocative studies by Tonegawa and Karayiorgou raise the possibility that calcineurin signaling plays a causative role in schizophrenia $[17,18]$. They performed association studies with calcineurin-related genes, prioritizing those located in schizophrenia susceptibility loci.

In their US and South African samples, allelic variants in the PPP3CC gene, encoding CaNA $\gamma$, showed preferential transmission from parents to affected offspring. In addition, conditional knockout mice lacking CaNB1 in the forebrain display traits of potential relevance to schizophrenia, such as deficits in working memory, diminished prepulse and latent inhibition and an increased response to the NMDAR antagonist MK801 [16,18].

These studies provided support for the idea that alterations in calcineurin function contribute to schizophrenia pathogenesis; however, no real causal variants in the PPP $3 C C$ gene were identified, and the mechanisms of aberrant signaling are still unknown.

In a recent study to explore the involvement of calcineurin dysfunction in schizophrenia, we tested for genetic association between a subset of calcineurin-related candidate genes and schizophrenia in Japanese cohorts [19]. Four of these genes (PPP3CC, EGR2, -3 and -4) showed significant association with schizophrenia.

'The turnover of genome-wide association studies will accelerate with the advance of high-density single nucleotide polymorphism chip arrays'

Moreover, in the prefrontal cortex of postmortem brains from schizophrenic patients, EGR1, -2 and -3 transcripts were downregulated. Our results provide confirmation for the previously reported genetic association of PPP3CC with schizophrenia, and suggest a potentially important role for the $E G R$ gene family (the family consists of EGR1-4 genes) in schizophrenia pathogenesis. These findings all support the theory of altered calcineurin signaling in schizophrenia.

Interestingly, $E G R 3$ is located in the same chromosomal region as $P P P 3 C C$, within $8 \mathrm{p} 21.3$, and is functionally linked to dopamine and glutamate signaling. This result exemplifies the idea that a single linkage region may contain multiple susceptibility genes, and that genes from different functional families can contribute to the linkage signal from a single region [7].

The EGR gene family is an important mediator of gene transcription in neuronal development and plasticity and apoptotic pathways. Notably, Egr2 and -3 are necessary for normal brain development. For instance, Egr2 (also known as Krox20) is indispensable in hindbrain patterning and peripheral nerve myelination [20,21]. Egr3 is also essential for the development of muscle spindles in mice [22,23]. 
In addition to their downstream position in calcineurin signaling, the EGR proteins interact with a number of other factors implicated in schizophrenia. Both EGR2 and EGR3 are downstream targets of NRG1 [24]. It is intriguing that defects in NRG1-ErbB (one of the NRG1 receptors) signaling may affect oligodendrocyte development and myelination, given that multiple lines of evidence converge to implicate abnormalities in oligodendroglia and myelin in schizophrenia [25,26]. Additionally, reports suggest that transcription of the $E G R$ genes can be regulated by the methylation status of $\mathrm{CpG}$ islands [27]. Therefore, epigenetic studies focusing on EGRs could provide invaluable information.

The genes, EGR2 and -3 are of particular interest as downstream targets for calcineurin in light of the reported close relationship between schizophrenia and autoimmune disease [28-30]. Egr2 and -3 are regulated by calcineurin through activation of nuclear factor of activated $\mathrm{T}$ cells (NFAT)-mediated transcription [31]. NFAT and Egr2/-3 are key molecules in T-cell activation [32]. Mice deprived of mature $\mathrm{T}$ cells are reported to manifest cognitive deficits and behavioral abnormalities consistent with those observed in schizophrenia [33].

Calcineurin is also linked to the Akt/glycogen synthase kinase (GSK)-3-signaling cascade, which is another proposed contributory factor in schizophrenia and a critical apoptosis regulator $[8]$.

\section{'While EGRs may have limited effects ... in combination with the other calcineurin-related genes and/or downstream effectors their role may be greatly enhanced.'}

Akt can induce apoptosis by a Fas liganddependent mechanism, in which the Fas ligand gene is regulated by a number of transcription factors, including NFAT and Egr3 [34]. In addition, GSK-3 antagonizes calcineurin signaling by phosphorylation of NFAT proteins [35].

Worthy of attention is the fact that expression of the entire family of EGRs can be regulated by muscarinic acetylcholine receptors, which in turn are altered in schizophrenia [36-40]. For instance, muscarinic receptors are decreased in the cortex of schizophrenic brains, and muscarinic acetylcholine receptor (M1 and -4) knockout mice display locomotor behavioral abnormalities $[41,42]$.
Taken together, altered EGR function could contribute to schizophrenia pathogenesis through a variety of mechanisms. A potential mechanistic relationship between EGRs and other candidate pathways is an issue for further investigation.

\section{Future perspective}

What would be a step in the right direction for genetic studies on schizophrenia? Until recently, susceptibility genes have been the center of attention in analyzing the genetics of schizophrenia. Hunting for susceptibility genes within linked chromosomal regions has proven to be practical and beneficial for understanding schizophrenia. $D T N B P 1$ and $N R G 1$ were identified from linkage studies and, recently, a paradigm shift from linkage to association analyses has become evident.

Turnover of genome-wide association studies will accelerate with the advance of high-density single nucleotide polymorphism chip arrays [43]. This could provide an opportunity to find unexpected novel genes as candidates. The main question now is how to evaluate the biological significance of these single genes in the context of the complex nature of schizophrenia? It is likely that they have a limited genetic effect and wide genetic heterogeneity. The considerable diversity of disease symptoms and courses may result from the different combinations of genetic substrates in different patients. Moreover, it is unclear whether the identified susceptibility genes are causal of the disease or just weak modifiers of symptoms and courses.

It is worth emphasizing that PPP $3 C C$ and $E G R s$ were discovered after the analysis of pathway-based signaling. Compared with classical approaches, focusing on a group of genes belonging to the same functional pathway could yield the best results. Even though genetic susceptibility is heterogeneous, particular constellations of genes in the pathway might efficiently capture a common pattern for most patients. Towards the ultimate end, these pathways are a target for the development of better therapeutics. Our calcineurin studies were designed to determine whether a gene or a signaling pathway would contribute to the genetic risk for schizophrenia. While EGRs may have limited effects on the development of schizophrenia on their own, in combination with the other calcineurin-related genes and/or downstream effectors their role may be greatly enhanced. Thus, it will be invaluable to unravel the genetic architecture of putative molecular disease pathways in future studies. Identification of specific pathways will also provide promising targets for therapy. 


\section{Bibliography}

1. Norton N, Williams HJ, Owen MJ: An update on the genetics of schizophrenia. Curr. Opin. Psychiatry 19, 158-164 (2006).

2. Owen MJ, Craddock N, O'Donovan MC: Schizophrenia: genes at last? Trends Genet. 21, 518-525 (2005).

3. Riley B, Kendler KS: Molecular genetic studies of schizophrenia. Eur. J. Hum. Genet. 14, 669-680 (2006).

4. Kirov G, O’Donovan MC, Owen MJ: Finding schizophrenia genes. J. Clin. Invest. 115, 1440-1448 (2005).

5. Karayiorgou M, Gogos JA: Schizophrenia genetics: uncovering positional candidate genes. Eur. J. Hum. Genet. 14, 512-519 (2006).

6. Gogos JA, Gerber DJ: Schizophrenia susceptibility genes: emergence of positional candidates and future directions. Trends Pharmacol. Sci. 27, 226-233 (2006).

7. Straub RE, Weinberger DR: Schizophrenia genes - famine to feast. Biol. Psychiatry 60, 81-83 (2006).

8. Emamian ES, Hall D, Birnbaum MJ, Karayiorgou M, Gogos JA: Convergent evidence for impaired AKT1-GSK3 $\beta$ signaling in schizophrenia. Nat. Genet. 36, 131-137 (2004).

9. Manji HK, Gottesman II, Gould TD: Signal transduction and genes-to-behaviors pathways in psychiatric diseases. Sci. STKE 2003, pe49 (2003).

10. Collier DA, Li T: The genetics of schizophrenia: glutamate not dopamine? Eur. J. Pharmacol. 480, 177-184 (2003).

11. Stefansson H, Sigurdsson E, Steinthorsdottir V et al:: Neuregulin 1 and susceptibility to schizophrenia. Am. J. Hum. Genet. 71, 877-892 (2002).

12. Straub RE, Jiang Y, MacLean CJ et al: Genetic variation in the $6 \mathrm{p} 22.3$ gene DTNBP1, the human ortholog of the mouse dysbindin gene, is associated with schizophrenia. Am. J. Hum. Genet. 71, 337-348 (2002).

13. Groth RD, Dunbar RL, Mermelstein PG: Calcineurin regulation of neuronal plasticity. Biochem. Biophys. Res. Commun. 311, 1159-1171 (2003).

14. Shibasaki F, Hallin U, Uchino $\mathrm{H}$ : Calcineurin as a multifunctional regulator. J. Biochem. (Tokyo) 131, 1-15 (2002).

15. Greengard P: The neurobiology of dopamine signaling. Biosci. Rep. 21 , 247-269 (2001).
16. Zeng $\mathrm{H}$, Chattarji $\mathrm{S}$, Barbarosie $\mathrm{M}$ et al: Forebrain-specific calcineurin knockout selectively impairs bidirectional synaptic plasticity and working/ episodic-like memory. Cell 107, 617-629 (2001).

17. Gerber DJ, Hall D, Miyakawa T et al:: Evidence for association of schizophrenia with genetic variation in the $8 \mathrm{p} 21.3$ gene, PPP3CC, encoding the calcineurin $\gamma$ subunit. Proc. Natl Acad. Sci. USA 100, 8993-8998 (2003).

18. Miyakawa T, Leiter LM, Gerber DJ et al.: Conditional calcineurin knockout mice exhibit multiple abnormal behaviors related to schizophrenia. Proc. Natl Acad. Sci. USA 100, 8987-8992 (2003).

19. Yamada K, Gerber DJ, Iwayama Y et al:: Genetic analysis of the calcineurin pathway identifies members of the EGR gene family, specifically $E G R 3$, as potential susceptibility candidates in schizophrenia. Proc. Natl Acad. Sci. USA 104, 2815-2820 (2007).

20. Warner LE, Mancias P, Butler IJ et al. Mutations in the early growth response 2 (EGR2) gene are associated with hereditary myelinopathies. Nat. Genet. 18, 382-384 (1998).

21. Warner LE, Svaren J, Milbrandt J, Lupski JR: Functional consequences of mutations in the early growth response 2 gene (EGR2) correlate with severity of human myelinopathies. Hum. Mol. Genet. 8, 1245-1251 (1999).

22. Tourtellotte WG, Keller-Peck C, Milbrandt J, Kucera J: The transcription factor Egr3 modulates sensory axon-myotube interactions during muscle spindle morphogenesis. Dev. Biol. 232, 388-399 (2001)

23. Tourtellotte WG, MilbrandT J: Sensory ataxia and muscle spindle agenesis in mice lacking the transcription factor Egr3. Nat. Genet. 20, 87-91 (1998).

24. Jacobson C, Duggan D, Fischbach G: Neuregulin induces the expression of transcription factors and myosin heavy chains typical of muscle spindles in cultured human muscle. Proc. Natl Acad. Sci. USA 101, 12218-12223 (2004)

25. Corfas G, Roy K, Buxbaum JD: Neuregulin 1-erbB signaling and the molecular/cellular basis of schizophrenia. Nat. Neurosci. 7, 575-580 (2004).
26. Michailov GV, Sereda MW, Brinkmann BG et al.: Axonal neuregulin-1 regulates myelin sheath thickness. Science 304, 700-703 (2004).

27. Unoki $M$, Nakamura Y: Methylation at CpG islands in intron 1 of $E G R 2$ confers enhancer-like activity. FEBS Lett. 554, 67-72 (2003).

28. Jones AL, Mowry BJ, Pender MP, Greer JM: Immune dysregulation and self-reactivity in schizophrenia: do some cases of schizophrenia have an autoimmune basis? Immunol. Cell Biol. 83, 9-17 (2005)

29. Eaton WW, Byrne M, Ewald $\mathrm{H}$ et al: Association of schizophrenia and autoimmune diseases: linkage of Danish national registers. Am. J. Psychiatry 163 , 521-528 (2006)

30. Kipnis J, Cardon M, Strous RD, Schwartz M: Loss of autoimmune T cells correlates with brain diseases: possible implications for schizophrenia? Trends Mol. Med. 12, 107-112 (2006)

31. Hildeman DA, Mitchell T, Kappler J, Marrack P: T cell apoptosis and reactive oxygen species. J. Clin. Invest. 111, 575-581 (2003).

32. Safford M, Collins S, Lutz MA et al.: Egr-2 and Egr-3 are negative regulators of T-cell activation. Nat. Immunol. 6, 472-480 (2005).

33. Kipnis J, Cohen H, Cardon M, Ziv Y, Schwartz M: T cell deficiency leads to cognitive dysfunction: implications for therapeutic vaccination for schizophrenia and other psychiatric conditions. Proc. Natl Acad. Sci. USA 101, 8180-8185 (2004).

34. Brunet A, Bonni A, Zigmond MJ et al: Akt promotes cell survival by phosphorylating and inhibiting a Forkhead transcription factor. Cell 96, 857-868 (1999).

35. Antos CL, McKinsey TA, Frey $\mathrm{N}$ et al. Activated glycogen synthase- $3 \beta$ suppresses cardiac hypertrophy in vivo. Proc. Natl Acad. Sci. USA 99, 907-912 (2002).

36. von der Kammer H, Mayhaus M, Albrecht C, Enderich J, Wegner M, Nitsch RM: Muscarinic acetylcholine receptors activate expression of the EGR gene family of transcription factors. J. Biol. Chem. 273, 14538-14544 (1998).

37. Sarter M, Nelson CL, Bruno JP: Cortical cholinergic transmission and cortical information processing in schizophrenia. Schizophr. Bull. 31, 117-138 (2005). 
38. Raedler TJ, Knable MB, Jones DW et al: In vivo determination of muscarinic acetylcholine receptor availability in schizophrenia. Am. J. Psychiatry 160, 118-127 (2003).

39. Dean B, Bymaster FP, Scarr E: Muscarinic receptors in schizophrenia. Curr. Mol. Med. 3 , 419-426 (2003).

40. Dean B, McLeod M, Keriakous D,

McKenzie J, Scarr E: Decreased muscarinic 1 receptors in the dorsolateral prefrontal cortex of subjects with schizophrenia. Mol. Psychiatry 7, 1083-1091 (2002)
41. Gerber DJ, Sotnikova TD, Gainetdinov RR, Huang SY, Caron MG, Tonegawa S: Hyperactivity, elevated dopaminergic transmission, and response to amphetamine in M1 muscarinic acetylcholine receptordeficient mice. Proc. Natl Acad. Sci. USA 98, 15312-15317 (2001).

42. Gomeza J, Zhang L, Kostenis E et al: Enhancement of D1 dopamine receptor-mediated locomotor stimulation in M4 muscarinic acetylcholine receptor knockout mice. Proc. Natl Acad. Sci. USA 96, 10483-10488 (1999).

43. Jorgenson E, Witte JS: A gene-centric approach to genome-wide association studies. Nat. Rev. Genet. 7, 885-891 (2006).

\section{Affiliations}

- Kazuo Yamada RIKEN Brain Science Institute, Laboratory for Molecular Psychiatry, Wako, Saitama 351-0198, Japan Tel.: +81484675968; Fax: +8148467 7462; k_yamada@brain.riken.jp

- Takeo Yoshikawa, MD, PhD RIKEN Brain Science Institute, Laboratory for Molecular Psychiatry, 2-1 Hirosawa, Wako-city, Saitama 351-0198, Japan Tel.: +81484675968

Fax: +81484677462 ; takeo@brain.riken.jp 